\title{
How whole-body vibration can help our COPD patients. Physiological changes at different vibration frequencies
}

This article was published in the following Dove Press journal: International Journal of COPD

\section{Eulogio Pleguezuelos ${ }^{1-3}$ \\ Paula Casarramona' \\ Lluis Guirao ${ }^{1,3}$ \\ Beatriz Samitier \\ Pilar Ortega 4 \\ Xavier Vila ${ }^{4}$ \\ Amin Del Carmen' \\ Laura Ovejero ${ }^{4}$ \\ Eva Moreno 5 \\ Noemi Serra ${ }^{5}$ \\ Manuel Gomís ${ }^{3}$ \\ Manuel V Garnacho- \\ Castaño 3 \\ Marc Miravitlles ${ }^{6}$ \\ 'Physical Medicine and Rehabilitation Department, Mataró Hospital, Barcelona, Spain; ${ }^{2}$ Department of Experimental Science and Healthcare, Faculty of Health Sciences, Universitat Pompeu Fabra, Barcelona, Spain; ${ }^{3}$ School of Sciences, Tecnocampus- School of Health Sciencies, Pompeu Fabra University, Mataro, Barcelona, Spain; ${ }^{4}$ Pneumology Department, Mataró Hospital, Barcelona, Spain; ${ }^{5}$ Physical Medicine and Rehabilitation Department, Hospitalet General Hospital, L'Hospitalet de Llobregat, Barcelona, Spain; ${ }^{6}$ Pneumology Department, Hospital Universitari Vall d'Hebron, Ciber de Enfermedades Respiratorias (CIBERES), Barcelona, Spain}

Correspondence: Eulogio Pleguezuelos Hospital de Mataró, c/ Cirera s/n, 08302. Mataro, Barcelona, Spain

Tel +349374l 7728

Fax +3493 74I 77I7

Email epleguezuelos@csdm.cat
Objective: Evaluate cardiac, metabolic, and ventilatory changes during a training session with whole-body vibration training (WBVT) with 3 different frequencies in patients with chronic obstructive pulmonary disease (COPD).

Methods: This was a prospective, interventional trial in outpatients with severe COPD. Participants performed 3 vertical WBVT sessions once a week using frequencies of $35,25 \mathrm{~Hz}$ and no vibration in squatting position (isometric). Cardiac, metabolic, and ventilator parameters were monitored during the sessions using an ergospirometer. Changes in oxygen pulse response $\left(\mathrm{VO}_{2} / \mathrm{HR}\right)$ at the different frequencies were the primary outcome of the study.

Results: Thirty-two male patients with a mean forced expiratory volume in 1 second of $39.7 \%$ completed the study. Compared to the reference of $35 \mathrm{~Hz}, \mathrm{VO}_{2} / \mathrm{HR}$ at no vibration was $10.7 \%$ lower $(P=0.005)$; however, no statistically significant differences were observed on comparing the frequencies of 35 and $25 \mathrm{~Hz}$. The median oxygen uptake $\left(\mathrm{VO}_{2}\right)$ at $25 \mathrm{~Hz}$ and no vibration was $9.43 \%$ and $13.9 \%$ lower, respectively, compared to that obtained at $35 \mathrm{~Hz}$ (both comparisons $P<0.0001)$. The median expiratory volume without vibration was $9.43 \%$ lower than the $\mathrm{VO}_{2}$ at the end of the assessment at $35 \mathrm{~Hz}$ vibration $(P=0.002)$.

Conclusion: Vertical WBVT training sessions show greater cardiac, metabolic, and respiratory responses compared with the squat position. On comparing the 2 frequencies used, we observed that the frequency of $35 \mathrm{~Hz}$ provides higher cardiorespiratory adaptation.

Keywords: COPD, cardioventilatory response, exercise, whole-body vibration

\section{Introduction}

Chronic obstructive pulmonary disease (COPD) is a chronic respiratory condition that is often associated with multiple comorbidities. ${ }^{1}$ Among the extrapulmonary manifestations, skeletal muscle weakness and emaciation are frequent and are associated with exercise intolerance and health status deterioration in COPD patients, regardless of the level of airway obstruction. ${ }^{2}$

Skeletal muscle dysfunction in COPD is characterized by a significant reduction in muscle force and resistance, which may be secondary to multiple mechanisms. ${ }^{3}$ This dysfunction may appear in relatively early phases of the disease and impair the symptoms and quality of life of patients. ${ }^{4,5}$

In recent years, there has been increasing interest in whole-body vibration training (WBVT) as a form of exercise, especially among the elderly. ${ }^{6,7} \mathrm{WBVT}$ is done on a platform that generates vertical sinusoidal vibrations, which stimulate muscle spindles and produce muscular contractions similar to tonic reflexes. ${ }^{8}$ 
Gloeckl et $\mathrm{al}^{9}$ were the first to publish improvements in the 6-minute walk test (6MWT) and sit-to-stand test in a COPD population following WBVT. Later, our group concluded that treatment with WBVT for 6 weeks increased functional capacity measured by the 6MWT but did not improve the strength of knee flexors and extensors assessed by an isokinetic test. ${ }^{10}$ An interesting result of our study was the reduction in oxygen saturation and a mean increase in heart rate during the course of vertical WBVT with $33 \mathrm{~Hz}{ }^{10}$ However, it was not clear whether the answers were dependent on the frequency of the vibration. To date, few studies have described cardiac and ventilatory physiological changes during WBVT sessions, and those that are available have conflicting results that may not be fully explained by differences in the protocol. ${ }^{10-12}$ Therefore, the current study was designed with the main objective of evaluating cardiac and ventilatory changes during a training session with the WBVT at 3 different frequencies in a severe COPD population in order to identify the optimal frequency for training in this population.

\section{Methods}

\section{Study design and population}

This prospective, interventional trial was conducted to determine the changes in cardiac and ventilatory parameters during a WBVT session at different treatment frequencies. Patients referred to the Physical Medicine and Rehabilitation Department of the Hospital of Mataró (Barcelona, Spain) from September 2016 to May 2017 were included if they fulfilled the following inclusion criteria: severe COPD defined as a postbronchodilator forced expiratory volume in 1 second/ forced vital capacity $\left(\mathrm{FEV}_{1} / \mathrm{FVC}\right)<0.7$ and $\mathrm{FEV}_{1}<50 \%$ predicted and stable disease defined as no exacerbation, and hospital admission or change in treatment in the previous 3 months. The exclusion criteria were other significant respiratory disease (bronchiectasis, lung fibrosis, asthma, active tuberculosis), active smoking, severe cardiovascular, neurological, and/or metabolic pathology that could interfere with the results, total hip, knee, or ankle arthroplasty, previous vitreous hemorrhage, need for oxygen therapy, severe alcoholism $(>80 \mathrm{~g} / \mathrm{d}$ ), and severe malnutrition (body mass index $[\mathrm{BMI}]<19 \mathrm{~kg} / \mathrm{m}^{2}$ ) to reduce the risk of falling and dropouts.

All patients performed forced spirometry. Respiratory muscle strength was assessed through maximum inspiratory pressure (MIP) and maximum expiratory pressure (MEP) evaluated using a Jaeger MasterScreen (Quebec, Canada) with a pressure transducer connected to the mouth in accordance with guidelines and recommended reference values. ${ }^{13}$
The BMI, obstruction, dyspnea, exercise index were calculated in all the participants. ${ }^{14}$ The $6 \mathrm{MWT}$ was used to assess exercise capacity and was performed following international recommendations. ${ }^{15}$ To determine the impact of the disease on quality of life the COPD assessment test was used. ${ }^{16}$ The Hospital Anxiety and Depression Scale was used to assess the psychological status of patients. ${ }^{17}$

The study was approved by the Research and Ethics Committee of the Hospital of Mataró, and all patients provided written informed consent. The study complies with the Spanish legislation for clinical studies, and registration is not mandatory.

\section{WBVT protocol}

Patients were invited to attend 3 sessions of WBVT at different vibration frequencies separated by 7 days. A vertical vibration platform Fitybe excel pro was used (GymnaUniphy Nv, Bilzen, Belgium). The training was designed to perform static work of the lower limbs. Patients worked in a squatting position, with $30^{\circ}$ of hip flexion and $55^{\circ}$ of knee flexion, holding onto the bars of the WBV platform. Each session consisted on 6 series of 430 -second repetitions with a frequency of $35 \mathrm{~Hz}$ in the first session, $25 \mathrm{~Hz}$ in the second session, and no vibration in the third session on consecutive weeks. In all the sessions the amplitude was $2 \mathrm{~mL}$, with 60 seconds of rest between each of the 6 series. The selection of frequencies was based on previous studies analyzing the effects at frequencies ranging from 24 to $35 \mathrm{~Hz} .{ }^{18}$ Patients wore a mask for respiratory gas collection and analyses throughout the test.

The intensity of the physical exercise was controlled by the patients, using the modified Borg scale. This scale was used during exercise to quantify the intensity of shortness of breath the patient was suffering. Difficulty in breathing was scored on a scale from 0 to 10 , with 0 representing "no shortness of breath" and 10 indicating "so much shortness of breath that the activity had to be stopped."19 Patients were informed as to the correct use of the scale to ensure that the mean value was not exceeded during the exercise.

Patients were continuously monitored by medical staff and a physiotherapist, who corrected the position on the platform and monitored outcome parameters as well as possible complications. The medical staff and the physiotherapist did not participate in the evaluation of the results.

\section{Outcomes}

The primary outcome of the study was oxygen pulse response $\left(\mathrm{VO}_{2} / \mathrm{HR}\right)$ in $\mathrm{mL} /$ beat at the end of the training session. 
All spiroergometric outcomes were evaluated at the end of the last exercise session.

Secondary outcomes were as follows: 1) cardiac changes as heart rate in beats per minute (bpm); 2) metabolic changes as in oxygen uptake $\left(\mathrm{VO}_{2}\right)$ in $\mathrm{mL} / \mathrm{min}$ and carbon dioxide output $\left(\mathrm{VCO}_{2}\right)$ in $\mathrm{mL} / \mathrm{min}$; and 3 ) respiratory changes as in tidal volume $\left(V_{\mathrm{t}}\right)$ in $\mathrm{mL}$, volume of expired gas $\left(V_{\mathrm{E}}\right)$ in $\mathrm{L} / \mathrm{min}$, and respiratory rate $(\mathrm{RR})$ in breaths per minute $(\mathrm{br} / \mathrm{min})$. All variables were measured at the end of the training session using the CPX Ergoespirometer System (Medical Graphics Corporation, Saint Paul, MN, USA).

\section{Statistical analysis}

Data are presented as mean \pm standard deviation (SD). All outcomes except heart rate presented a skewed distribution and were log-transformed to approximate normality. Random-effects linear mixed models with repeated measures were run to evaluate the effect of vibration frequency (3 levels: no vibration, $25 \mathrm{~Hz}$ of vibration frequency, $35 \mathrm{~Hz}$ of vibration frequency) on outcomes at the end of the training session. Models were adjusted for the baseline value of the outcome response. For heart rate, adjusted means and coefficients (Coef) with 95\% confidence intervals (CIs) were presented. For the rest of the outcomes, adjusted geometric means (or medians) and the relative change (calculated as $\left.100 *\left(\mathrm{e}^{\text {Coef }}-1\right) \%\right)$ with $95 \%$ CI were presented.

\section{Results}

\section{Population}

Thirty-five patients were recruited for the study, but 2 did not attend the appointments and 1 required hospital admission for an exacerbation during the study period. Therefore, the final population consisted of 32 patients, all of whom were males. Their mean age (SD) was 68.1 (9.9) years, with a mean BMI of $26.3(4.6) \mathrm{kg} / \mathrm{m}^{2}$, and a mean $\mathrm{FEV}_{1} \%$ of $39.7 \%(3.4 \%$ ) (Table 1 ).

\section{Main outcome: oxygen pulse}

A progressive increase in $\mathrm{VO}_{2} / \mathrm{HR}$ was observed with the increase in the frequency of vibration. The $\mathrm{VO}_{2} / \mathrm{HR}$ at $0 \mathrm{~Hz}$ was $5.69 \mathrm{~mL} /$ beat, which increased to $5.93 \mathrm{~mL} /$ beat at $25 \mathrm{~Hz}$ and to $6.38 \mathrm{~mL} /$ beat at $35 \mathrm{~Hz}$ (Figure 1). Compared to the reference of $35 \mathrm{~Hz}, \mathrm{VO}_{2} / \mathrm{HR}$ at no vibration was $10.7 \%$ lower $(P=0.005)$; however, the decrease in $\mathrm{VO}_{2} / \mathrm{HR}$ between 35 and $25 \mathrm{~Hz}$ was not statistically significant (Table 2).

\section{Secondary outcomes}

Statistically significant differences were observed in heart rates between $35 \mathrm{~Hz}$ and no vibration $(-4.39 \%$ less; $P<0.05)$,
Table I Demographic, clinical, and descriptive functional characteristics at baseline

\begin{tabular}{lll}
\hline $\begin{array}{l}\text { Characteristics of patients } \\
\text { with COPD (n=32) }\end{array}$ & Mean & SD \\
\hline Anthropometric variables & & \\
Age, years & 68.1 & 6.9 \\
BMI, kg/m & 26.3 & 4.1 \\
Lung function & & \\
FVC, \% & 61.8 & 11.9 \\
FEV,$\%$ & 39.7 & 3.4 \\
FEV,/FVC, \% & 48.6 & 15.7 \\
MIP (cm $\left.\mathrm{H}_{2} \mathrm{O}\right), \%$ & 68.5 & 14,1 \\
MEP (cm H $\left.\mathrm{H}_{2} \mathrm{O}\right), \%$ & 95.4 & 20.2 \\
Functional capacity, 6MWT & & \\
Meters walked & 347 & 35 \\
Maximum oxygen desaturation, \% & 89.7 & 5.0 \\
Borg scale & 8.4 & 1.2 \\
BODE index score & 4.5 & 1.3 \\
CAT score & 15.3 & 7.2 \\
Anxiety HADS & 7.1 & 3.4 \\
Depression HADS & 8.5 & 2.4 \\
\hline
\end{tabular}

Abbreviations: 6MWT, 6-minute walk test; $\mathrm{BMI}$, body mass index; BODE, BMI, obstruction, dyspnea exercise; CAT, COPD assessment test; COPD, chronic obstructive pulmonary disease; $\mathrm{FEV}_{\mid}$, forced expiratory volume in I second; FVC, forced vital capacity; HADS, Hospital Anxiety and Depression Scale; MEP, maximum expiratory pressure; MIP, maximum inspiratory pressure; SD, standard deviation.

but not between 35 and $25 \mathrm{~Hz}$. Regarding metabolic variables (Figure 2), the median $\mathrm{VO}_{2}$ at the end of the assessment at both no vibration and $25 \mathrm{~Hz}$ was significantly lower than that obtained at the reference frequency of $35 \mathrm{~Hz}$ (Table 2). In contrast, there were no significant differences between frequencies in $\mathrm{VCO}_{2}$.

At a ventilatory level (Figure 3), no significant differences were observed in $V_{\mathrm{t}}$, but the median $V_{\mathrm{E}}$ at the end of the assessment at both no vibration and $25 \mathrm{~Hz}$ was significantly lower than that measured at $35 \mathrm{~Hz}$. No differences were observed in RR at 0 and $25 \mathrm{~Hz}$; however, there was a statistically significant increase in the RR compared with the results at $35 \mathrm{~Hz}$ compared to no vibration and $25 \mathrm{~Hz}$ $(P<0.05)$ (Table 2).

\section{Safety}

There were no adverse events during the assessment of the patients at any of the 3 frequencies tested.

\section{Discussion}

Our results show that there is a significant increase in oxygen pulse during the course of a training session with WBVT compared to isometric squat exercises in COPD patients. Changes were also found in the metabolic and ventilatory parameters at 25 and $35 \mathrm{~Hz}$ compared to the assessment at no vibration. According to these results, $35 \mathrm{~Hz}$ appears to 

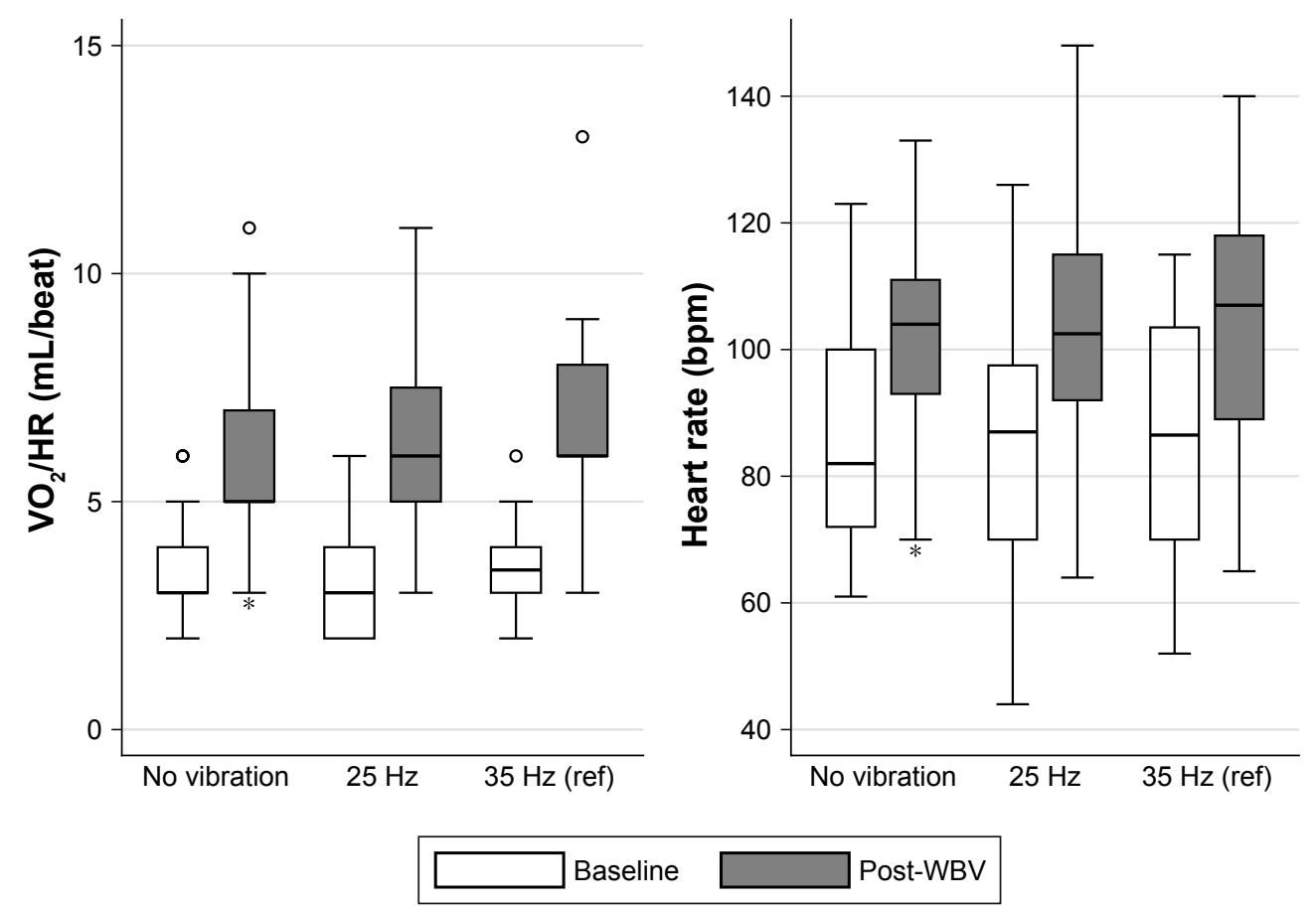

Figure I Description of cardiac variables at rest and at different training frequencies.

Notes: The $\mathrm{VO}_{2} / \mathrm{HR}$ in $\mathrm{mL} /$ beat, cardiac or heart rate in bpm. ref $=$ reference category; ${ }^{*} P$-value $<0.05$ versus $35 \mathrm{~Hz}$ (ref).

Abbreviations: $\mathrm{VO}_{2} / \mathrm{HR}$, oxygen pulse response; WBV, whole-body vibration.

be the optimal vibration frequency for training in severe COPD patients.

In recent years, WBVT has become a popular form of physical exercise. WBVT induces a tonic vibration reflex, which produces involuntary monosynaptic muscle contractions, especially in the lower extremities. ${ }^{20}$ Several studies have described improvement in exercise capacity, postural control, and muscle power, ${ }^{9,10,21,22}$ with no improvement in muscle strength. ${ }^{9,22}$ The mechanisms of these benefits remain unclear. However, they may be due to multifactorial causes, in which improvements at the level of the neuromuscular system ${ }^{22}$ and cardiorespiratory adaptations ${ }^{10}$ predominate. In a systematic review by Gloeckl et al, ${ }^{18}$ it was concluded that training with WBVT shows promising preliminary efficacy in patients in COPD; however, the number of studies is still limited and there is some heterogeneity in the variables of prescription of training.

A previous study by our group was the first to demonstrate an average increase of $15.8 \mathrm{bpm}$ in heart rate from the beginning to the end of the training session performed with a frequency of $35 \mathrm{~Hz}$, suggesting cardiovascular system variations with WBVT. ${ }^{10}$ However, more recent studies

Table 2 Differences in the cardiac, metabolic, and ventilatory variables in relation to the frequency of training with the vibratory platform

\begin{tabular}{|c|c|c|c|c|c|}
\hline \multirow[t]{3}{*}{ Variables } & \multicolumn{3}{|l|}{ WBV } & \multicolumn{2}{|l|}{ Difference } \\
\hline & $\mathbf{0 ~ H z}$ & $25 \mathrm{~Hz}$ & $35 \mathrm{~Hz}$ (ref) & 25 vs $35 \mathrm{~Hz}$ & 0 vs $35 \mathrm{~Hz}$ \\
\hline & $\operatorname{Mean}^{a}(95 \% \mathrm{Cl})$ & $\operatorname{Mean}^{\mathrm{a}}(95 \% \mathrm{Cl})$ & $\operatorname{Mean}^{\mathrm{a}}(95 \% \mathrm{Cl})$ & Change $^{\mathrm{b}}(95 \% \mathrm{Cl})$ & Change $^{\mathrm{b}}(95 \% \mathrm{Cl})$ \\
\hline $\mathrm{VO}_{2} / \mathrm{HR}$ (mL/beat) & $5.69(5.08,6.38)$ & $5.93(5.31,6.62)$ & $6.38(5.71,7.12)$ & $-7.01 \%(-13.94,0.47)$ & $-10.72 \% *(-17.83,-2.98)$ \\
\hline Heart rate (bpm) & $99.6(95.1,104.1)$ & $101.5(97.1,105.8)$ & $104.0(99.6,108.3)$ & $-2.5 \mathrm{I}(-5.47,0.44)$ & $-4.39 *(-7.53,-1.24)$ \\
\hline $\mathrm{VO}_{2}(\mathrm{~mL} / \mathrm{min})$ & $563.5(5 \mid 3.4,618.5)$ & $591.3(540.0,647.3)$ & $65 \mid .5(594.9,7 \mid 3.4)$ & $-9.24 \% *(-14.56,-3.59)$ & $-13.5 \% * *(-18.84,-7.81)$ \\
\hline $\mathrm{VCO}_{2}(\mathrm{~mL} / \mathrm{min})$ & $603.4(546.7,665.9)$ & $605.3(550.5,665.6)$ & $633.4(576.1,696.5)$ & $-4.44 \%(-11.29,2.94)$ & $-4.75 \%(-11.99,3.1)$ \\
\hline$V_{\mathrm{t}} \mathrm{BTPS}(\mathrm{mL})$ & I,036.6 (954.5, I,I I25.7) & I,060.3 $(978.8, I, \mid$ I48.6) & I,059.4 (977.8, I,I47.7) & $0.09 \%(-5.39,5.89)$ & $-2.15 \%(-7.82,3.87)$ \\
\hline$V_{\mathrm{E}} \mathrm{BTPS}(\mathrm{L} / \mathrm{min})$ & $25.1(22.7,27.8)$ & $25.3(22.9,27.9)$ & $27.8(25.2,30.6)$ & $-8.89 \% *(-|4.2|,-3.24)$ & $-9.43 \% *(-15.05,-3.45)$ \\
\hline $\mathrm{RR}(\mathrm{br} / \mathrm{min})$ & $24.3(22.0,26.9)$ & $23.91(21.6,26.4)$ & $26.0(23.6,28.7)$ & $-8.29 \% *(-13.03,-3.28)$ & $-6.55 \% *(-11.73,-1.08)$ \\
\hline
\end{tabular}

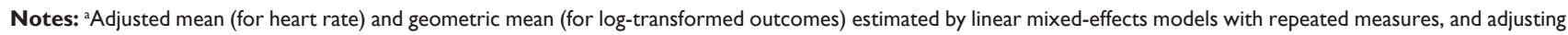
for baseline values; ' coefficient (for heart rate) or relative change; $* P<0.05 ; * * P<0.00$ I.

Abbreviations: br/min, breaths per minute; $\mathrm{BTPS}$, body temperature and pressure saturated; $\mathrm{Cl}$, confidence interval; $\mathrm{RR}$, respiratory rate; $\mathrm{VCO}$, carbon dioxide output; $V_{E}$, volume of expired gas; $\mathrm{VO}_{2}$, oxygen uptake; $\mathrm{VO}_{2} / \mathrm{HR}$, oxygen pulse response; $V_{\mathrm{t}}$, tidal volume; $\mathrm{WBV}$, whole-body vibration. 

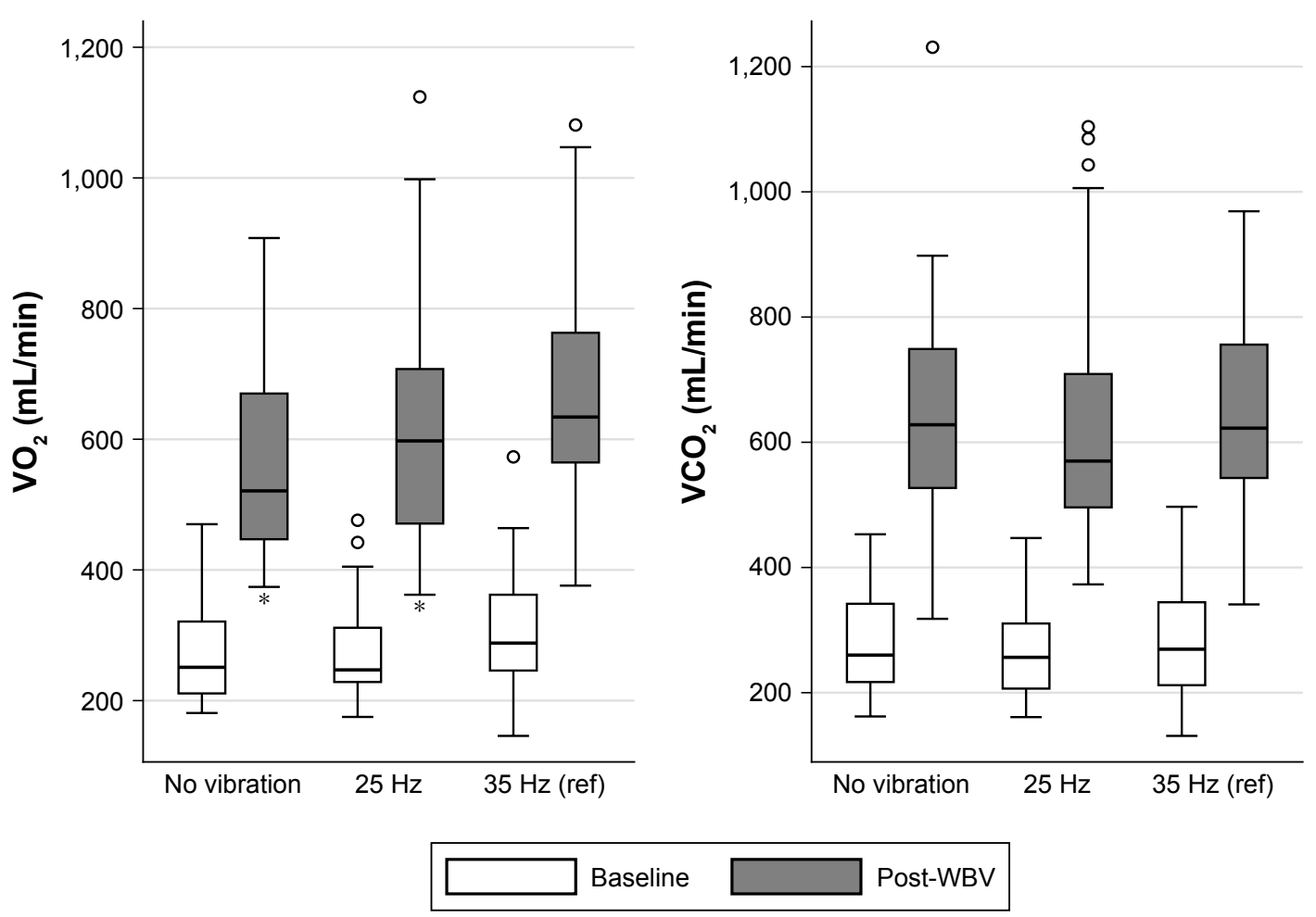

Figure 2 Description of metabolic variables at rest and at different training frequencies.

Notes: $\mathrm{VO}_{2}$ in $\mathrm{mL} / \mathrm{min}$ and $\mathrm{VCO}_{2}$ in $\mathrm{mL} / \mathrm{min}$. ref = reference category; $* P$-value $<0.05$ versus $35 \mathrm{~Hz}$ (ref).

Abbreviations: $\mathrm{VCO}_{2}$, carbon dioxide output; $\mathrm{VO}_{2}$, oxygen uptake; WBV, whole-body vibration.
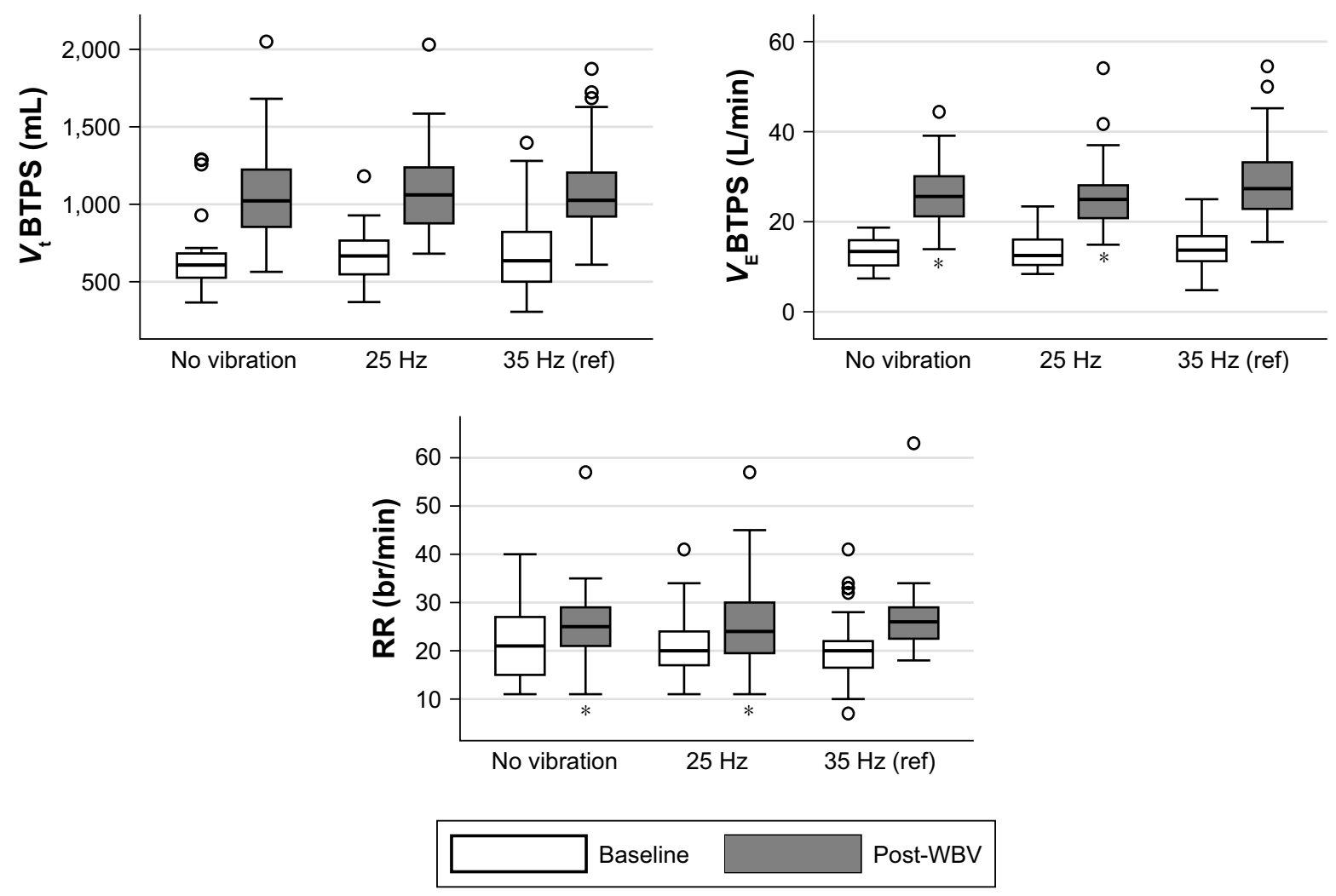

Figure 3 Description of ventilatory variables at rest and at different training frequencies.

Notes: $V_{t}$ in $\mathrm{mL}, V_{\mathrm{E}}$ in $\mathrm{L} / \mathrm{min}$, and $\mathrm{RR}$, in breathing per minute $(\mathrm{br} / \mathrm{min})$. ref = reference category; ${ }^{*} P$-value $<0.05$ versus $35 \mathrm{~Hz}$ (ref).

Abbreviations: $b r / m i n$, breaths per minute; $R R$, respiratory rate; $V_{\mathrm{E}}$, volume of expired gas; $V_{\mathrm{t}}$, tidal volume; WBV, whole-body vibration. 
have shown contradictory results in terms of the possible cardiorespiratory adaptations to WBVT. Therefore, the primary outcome of the present study was to evaluate oxygen pulse $\left(\mathrm{VO}_{2} / \mathrm{HR}\right)$ because this is a good surrogate of cardiac output during exercise. ${ }^{23}$ Two different training frequencies with the squat exercise ( $0 \mathrm{~Hz}$ frequency) were compared in order to verify cardiac adaptation with the WBVT and to identify the frequency that provides the best training for patients with COPD.

The oxygen pulse observed at $35 \mathrm{~Hz}$ was a median of $10.7 \%$ higher than that obtained at no vibration and was also $7 \%$ higher than that registered with training at $25 \mathrm{~Hz}$, although in this case the differences were not statistically significant. These results confirm that vibration training increases cardiac output in a dose-dependent fashion and may explain why the blood flow in the muscle is reduced in proportion to the percentage of maximum force during static or isometric exercise (ie, squat position). To counteract this reduction in blood flow, cardiac output, sympathetic activity, and the mean blood pressure increased. One possible hypothesis for the results obtained in the present study is that this previously described physiological adaptation may be increased by the effect of the vibration in the lower extremities and the sum of the vibration of the musculature of the upper extremities. Thus, this increase in cardiac output might explain part of the improvement in the exercise capacity of COPD patients obtained with WBVT.

Similar to our previous study, ${ }^{10}$ a slight, progressive increase in heart rate was observed with the squat position at the vibration frequencies of 25 and $35 \mathrm{~Hz}$, with differences between no vibration and $35 \mathrm{~Hz}$ reaching statistical significance. Nevertheless, 2 other studies did not observe a statistically significant difference between the increases in heart rate obtained after a session of WBVT or with the squat position. ${ }^{11,12}$ These contradictory results can be explained by differences in the training protocol (dynamic vs static protocol) or the different sample size of the studies.

The $\mathrm{VO}_{2}$ significantly and progressively increased with the 3 training frequencies used. In agreement with these results, previous studies in healthy volunteers have demonstrated a significant increase in $\mathrm{VO}_{2}$ with WBVT. ${ }^{24,25}$ Regarding COPD patients, Gloeckl et al ${ }^{12}$ also demonstrated a significant increase in $\mathrm{VO}_{2}$ on comparing training with and without vibration in a group of 10 patients with severe COPD; however, differences in $\mathrm{VO}_{2}$ between sessions with and without vibration were just under statistical significance. In the 1-minute session vibration increased $\mathrm{VO}_{2}$ by $6.8 \%$ $(P=0.074)$, by $5.02 \%$ in the 2 -minute session $(P=0.093)$, and by $6.79 \%$ in the 3 -minute session $(P=0.093)$. The lack of significance might be due to the small sample size of the study. Currently, the adaptive mechanisms that explain an increase in $\mathrm{VO}_{2}$ produced by training with vibrating platform are not very clear. We suspect that these increases in $\mathrm{VO}_{2}$ could be due to improvements in the peripheral components of the cardiovascular system and to an increase in blood flow in the active skeletal muscle produced by the vibration. In turn, training with WBVT could increase muscle mass and cause a greater recruitment of previously inactive motor units, which would result in a higher RR. ${ }^{26-28}$

We also found a progressive and statistically significant increase in $V_{\mathrm{E}}$ with an increasing frequency of vibration. Similarly, a previous study found an increase in $V_{\mathrm{E}}$ in the session with $26 \mathrm{~Hz}$ compared with the squat exercise, although the differences were just below significance $(P=0.052){ }^{12}$ The RR increased by $6.5 \%$ only at the frequency of $35 \mathrm{~Hz}$, but no differences were observed between no vibration and $25 \mathrm{~Hz}$. In contrast, the $V_{\mathrm{t}}$ progressively increased, albeit not significantly, with the increase in training frequency. This respiratory adaptation appears to be different from that observed in the previous study by Gloeckl et al ${ }^{12}$ who observed a $20 \%$ increase in the RR in the 3-minute training session with vibration compared to the same training without vibration. The increase in RR observed in the previous study and in our session at $35 \mathrm{~Hz}$ may be related to the increase in physiological demand produced during more intense physical exercise and may also be associated with the stimulation of motor units which are inactive at the level of the respiratory muscles as well as an increase in involuntary respiration by neural inspiratory stimulation. ${ }^{29}$

Gloeckl et al $^{12}$ used a side-alternating platform, while our results were obtained with the use of a vertical platform. These data should be taken into account on comparing the results, because the frequencies may not be comparable.

\section{Limitations}

The present study has some limitations. The study population was composed of only males, which is consistent with the epidemiology of severe COPD in Spain, ${ }^{30}$ but extrapolation of our results to women should be made with caution. Patients requiring oxygen therapy were excluded from the study, and therefore the results obtained can only be applied to patients not requiring this therapy. Furthermore, due to the lack of previous estimates of the effect and the exploratory characteristic of our study, a calculation of sample size was not made. However, our sample was larger than most of the studies published analyzing cardiorespiratory response during 
WBVT training and was large enough to demonstrate significant differences in outcomes between different vibration frequencies. We consider that the non-randomization of the sessions could be another limitation of the study, as well as the lack of familiarization with the WBVT on the part of the patients. However, among the strengths of our study, a trained, qualified person controlled the position of the patients during exercise, thereby avoiding possible bias in the squat position with and without vibration. Moreover, the squat position was performed without vibration on the turned off platform in order to minimize any bias of position.

\section{Conclusion}

The results of the present study show that the response in cardiac, metabolic, and ventilatory parameters during vertical WBVT training sessions may be greater compared with squat exercise. These responses may, in part, produce the improvements in exercise capacity observed after WBVT training programs. Although greater changes in the study parameters were observed at a vibration frequency of $25 \mathrm{~Hz}$ compared to training without vibration, the present results demonstrate that a vibration frequency of $35 \mathrm{~Hz}$ significantly increases response and may be associated with greater improvements in functional capacity. In addition, WBVT was safe and well tolerated. Further studies are needed to confirm these results and establish the most adequate frequency, amplitude, and duration of WBVT in different populations of patients with COPD with different degrees of severity.

\section{Disclosure}

Marc Miravitlles received speaker fees from Boehringer Ingelheim, Chiesi, Cipla, Menarini, Rovi, Bial, CSL Behring, Grifols and Novartis, consulting fees from Boehringer Ingelheim, Chiesi, GlaxoSmithKline, Bial, Gebro Pharma, CSL Behring, Laboratorios Esteve, Mereo Biopharma, Verona Pharma, pH Pharma, Novartis and Grifols and research grants from GlaxoSmithKline and Grifols, all outside of the submitted work. The authors report no other conflicts of interest in this work.

\section{References}

1. Miravitlles M, Price D, Rabe KF, Schmidt H, Metzdorf N, Celli B. Comorbidities of patients in tiotropium clinical trials: comparison with observational studies of patients with chronic obstructive pulmonary disease. Int J Chron Obstruct Pulmon Dis. 2015;10:549-564.

2. Matkovic Z, Cvetko D, Rahelic D, et al. Nutritional status of patients with chronic obstructive pulmonary disease in relation to their physical performance. COPD. 2017;14(6):626-634.

3. Pleguezuelos E, Esquinas C, Moreno E, et al. Muscular dysfunction in COPD: systemic effect or deconditioning? Lung. 2016;194(2): 249-257.
4. Barreiro E, Gea J. Respiratory and limb muscle dysfunction in COPD. COPD. 2015;12(4):413-426.

5. Gea J, Agustí A, Roca J. Pathophysiology of muscle dysfunction in COPD. J Appl Physiol. 2013;114(9):1222-1234.

6. Park SY, Son WM, Kwon OS. Effects of whole body vibration training on body composition, skeletal muscle strength, and cardiovascular health. J Exerc Rehabil. 2015;11(6):289-295.

7. Lienhard K, Vienneau J, Nigg S, Friesenbichler B, Nigg BM. Older adults show higher increases in lower-limb muscle activity during whole-body vibration exercise. J Biomech. 2017;52:55-60.

8. Burke D, Schiller HH. Discharge pattern of single motor units in the tonic vibration reflex of human triceps surae. J Neurol Neurosurg Psychiatry. 1976;39(8):729-741.

9. Gloeckl R, Heinzelmann I, Baeuerle S, et al. Effects of whole body vibration in patients with chronic obstructive pulmonary disease - a randomized controlled trial. Respir Med. 2012;106(1):75-83.

10. Pleguezuelos E, Pérez ME, Guirao L, et al. Effects of whole body vibration training in patients with severe chronic obstructive pulmonary disease. Respirology. 2013;18(6):1028-1034.

11. Furness T, Joseph C, Welsh L, Naughton G, Lorenzen C, et al. Whole-body vibration as a mode of dyspnoea free physical activity: a community-based proof-of-concept trial. BMC Res Notes. 2013; $6(1): 452$.

12. Gloeckl R, Richter P, Winterkamp S, et al. Cardiopulmonary response during whole-body vibration training in patients with severe COPD. ERJ Open Res. 2017;3(1):00101-2016.

13. Morales P, Sanchis J, Cordero PJ, Díez JL. Presiones respiratorias estáticas máximas en adultos. Valores de referencia de una población caucasiana mediterránea. [Maximum static respiratory pressures in adults. The reference values for a Mediterranean Caucasian population]. Arch Bronconeumol. 1997;33(5):213-219. Spanish.

14. Celli BR, Cote CG, Marín JM, et al. The body-mass index, airflow obstruction, dyspnea, and exercise capacity index in chronic obstructive pulmonary disease. N Engl J Med. 2004;350(10):1005-1012.

15. ATS Committee on Proficiency Standards for Clinical Pulmonary Function Laboratories. ATS statement: guidelines for the six-minute walk test. Am J Respir Crit Care Med. 2002;166(1):111-117.

16. Jones PW, Harding G, Berry P, Wiklund I, Chen WH, Kline Leidy N. Development and first validation of the COPD Assessment Test. Eur Respir J. 2009;34(3):648-654.

17. Herrero MJ, Blanch J, Peri JM, De Pablo J, Pintor L, Bulbena A. A validation study of the hospital anxiety and depression scale (HADS) in a Spanish population. Gen Hosp Psychiatry. 2003;25(4): 277-283.

18. Gloeckl R, Heinzelmann I, Kenn K. Whole body vibration training in patients with COPD: a systematic review. Chron Respir Dis. 2015;12(3): 212-221.

19. Borg G. Perceived exertion as an indicator of somatic stress. Scand J Rehabil Med. 1970;2(2):92-98.

20. Ritzmann R, Kramer A, Gruber M, Gollhofer A, Taube W. EMG activity during whole body vibration: motion artifacts or stretch reflexes? Eur J Appl Physiol. 2010;110(1):143-151.

21. Spielmanns M, Boeselt T, Gloeckl R, et al. Low-volume whole-body vibration training improves exercise capacity in subjects with mild to severe COPD. Respir Care. 2017;62(3):315-323.

22. Gloeckl R, Jarosch I, Bengsch U, et al. What's the secret behind the benefits of whole-body vibration training in patients with COPD? A randomized, controlled trial. Respir Med. 2017;126:17-24.

23. Stringer WW, Hansen JE, Wasserman K. Cardiac output estimated noninvasively from oxygen uptake during exercise. J Appl Physiol. 1997; 82(3):908-912.

24. Rittweger J, Ehrig J, Just K, Mutschelknauss M, Kirsch KA, Felsenberg D. Oxygen uptake in whole-body vibration exercise: influence of vibration frequency, amplitude, and external load. Int J Sports Med. 2002;23(6):428-432.

25. Rittweger J, Schiessl H, Felsenberg D. Oxygen uptake during wholebody vibration exercise: comparison with squatting as a slow voluntary movement. Eur J Appl Physiol. 2001;86(2):169-173. 
26. Kerschan-Schindl K, Grampp S, Henk C, et al. Whole-body vibration exercise leads to alterations in muscle blood volume. Clin Physiol. 2001;21(3):377-382.

27. Bogaerts A, Delecluse C, Claessens AL, Coudyzer W, Boonen S, Verschueren SM. Impact of whole-body vibration training versus fitness training on muscle strength and muscle mass in older men: a 1-year randomized controlled trial. J Gerontol A Biol Sci Med Sci. 2007;62(6): $630-635$.

28. Gerhardt F, Dumitrescu D, Gärtner C, et al. Oscillatory whole-body vibration improves exercise capacity and physical performance in pulmonary arterial hypertension: a randomised clinical study. Heart. 2017; 103(8):592-598.
29. Sumners DP, Green DA, Mileva KN, Bowtell JL. Increases in inspiratory neural drive in response to rapid oscillating airflow braking forces (vibration). Respir Physiol Neurobiol. 2008;160(3):350-352.

30. Miravitlles M, Soriano JB, García-Río F, et al. Prevalence of COPD in Spain: impact of undiagnosed COPD on quality of life and daily life activities. Thorax. 2009;64(10):863-868.

\section{Publish your work in this journal}

The International Journal of COPD is an international, peer-reviewed journal of therapeutics and pharmacology focusing on concise rapid reporting of clinical studies and reviews in COPD. Special focus is given to the pathophysiological processes underlying the disease, intervention programs, patient focused education, and self management protocols.

\section{Dovepress}

This journal is indexed on PubMed Central, MedLine and CAS. The manuscript management system is completely online and includes a very quick and fair peer-review system, which is all easy to use. Visit http://www.dovepress.com/testimonials.php to read real quotes from published authors.

Submit your manuscript here: http://www.dovepress.com/international-journal-of-chronic-obstructive-pulmonary-disease-journal 